\title{
LA DOCUMENTACION EN LAS INSTITUCIONES DE LA COMUNIDAD EUROPEA. UNA APROXIMACION BAJO LA PERSPECTIVA DEL TRATADO DE MAASTRICHT
}

\section{Juan Rodríguez Bouyssi *}

\section{Introducción}

Desde el día 7 de febrero de 1992, fecha en que los ministros de Asuntos Exteriores de los doce países integrantes de la Comunidad Europea firmaron el nuevo Tratado de la Unión Europea, popularmente conocido como Tratado de Maastricht, muchos han sido los análisis, opiniones, evaluaciones e interpretaciones que su texto ha suscitado. Es evidente que los acontecimientos políticos de los últimos meses han relanzado una fuerte discusión de fondo sobre la estructura y funcionamiento de la Comunidad Europea, no tanto por lo que es hoy en día, por su realidad actual, cuanto por los principios en que debe apoyarse para su evolución en su próximo futuro.

Posiblemente, el momento de las definiciones ha llegado. Es necesario, y urgente, diseñar la Europa que queremos construir tanto en sus contenidos (competencias comunitarias y principio de subsidiaridad) como en la forma que se debe dar a sus continentes (estructura institucional y funcional). El llamado «nuevo orden internacional» ha impulsado definitivamente este proceso, pues no sólo es necesaria una Europa más cohesionada que pueda hacer frente a sus relaciones económico-comerciales con las grandes potencias mundiales, sino también es necesaria una Europa más solidaria que dé una respuesta efectiva a aquellos países que, por razones de vecindad y de origen común, reclaman el nivel de desarrollo y de bienestar social que hoy representa la Comunidad Europea.

No es de extrañar, por tanto, que el Tratado de Maastricht no haya pasado inadvertido. Su texto ha despertado las fobias/filias en algunos paises, en que por estrecha mayoría ha sido rechazado/aceptado en referéndum popular, y es que, a diferencia del Acta Unica Europea, cuyos principales objetivos fueron delimitar los plazos definitivos en que debía terminarse la labor comenzada en 1957 con el Tratado de Roma, donde ya se definía el Mercado Unico, y paralelamente consolidar ciertas competencias de actuación de la CE ya iniciadas anteriormente, el nuevo Tratado de la Unión Europea diseña, en la práctica, lo que puede ser el germen de la Europa que habrá que construir en los años próximos: la Ciudadanía Europea; la Unión Económica y Monetaria; la Política Exterior y de Seguridad; etcétera. Es cierto, por tanto, que dicho tratado conlleva una importante carga de novedades cuyo fin último es, ni más ni menos, comenzar a dar respuestas a las cuestiones que hoy se plantean sobre los mecanismos de construcción de la Europa unida.

Ante este panorama, no renunciamos a sumarnos a los intentos de aproximación a la realidad comunitaria con ayuda del hilo conductor que nos ofrece el Tratado de Maastricht. En esta ocasión, lo haremos ayudados también por nuestra

* Oficina del Parlamento Europeo en España.

Recibido: 10-1-93.

Este texto es responsabilidad exclusiva de su autor y no compromete la opinión del Parlamento Europeo. 
óptica profesional: los sistemas y servicios de información y documentación. Con esta perspectiva, pretendemos dar respuesta a dos preguntas de base: ¿Responden los sistemas de información y documentación de la $\mathrm{CE}$ a las actuales demandas de publicidad de sus actividades? ¿Cuál será la incidencia de los mandatos del Tratado de Maastricht sobre dichos sistemas?

Esperamos, a lo largo de estas páginas, ofrecer un nuevo punto de vista, netamente profesional, eso sí, sobre los tan debatidos contenidos del Tratado de la Unión Europea.

\section{Sistemas de información y documentación de la $\mathrm{CE}$, un paso más hacia la publicidad y transparencia de su actuación}

En el más reciente Repertorio de Bases de Datos públicas de la $\mathrm{CE}$, editado con motivo de la Expo-92 de Sevilla, se recoge la información correspondiente a un total de cuarenta y dos bases y bancos de datos cuyo origen son las propias instituciones comunitarias, y los programas financiados directamente mediante fondos de la CE. Sería un grave error evaluar la capacidad de información tomando como base solamente la cantidad de sistemas que hoy dia se ofrecen. Sin embargo, basta esa cifra para comprender que uno de los principios rectores de la actuación de las instituciones de la $\mathrm{CE}$ ha sido el desarrollo de servicios avanzados de información y documentación, con el fin primordial de extender la publicidad de sus trabajos hasta los más apartados rincones de la geografía europea. No olvidemos que, a pesar de que los lugares habituales de trabajo de las instituciones comunitarias son Bruselas, Luxemburgo y Estrasburgo, éstas deben mantener un estrecho contacto tanto con las administraciones nacionales de los Estados miembros como con las entidades físicas y jurídicas de los mismos.

Por tanto, podemos apuntar un primer rasgo que caracteriza la información comunitaria: la diversidad y proliferación de servicios de información automatizada, con origen en la propia estructura institucional de la CE. Sin embargo, no nos debe preocupar tanto la cantidad como la calidad de estos servicios. Entendemos calidad, en este contexto, como la capacidad de responder a las verdaderas necesidades de información que los usuarios europeos puedan demandar. En definitiva, la adecuación del producto a las exigencias del mercado.

En este sentido, la experiencia nos demuestra que cada vez se está más cerca de cubrir, mediante sistemas automatizados, casi todos los requerimientos de información que se nos plantean. Especialmente en los dos últimos años, el avance ha sido sustancial tanto en la cobertura como en la calidad de los sistemas a nuestra disposición.

Tomemos como ejemplo el ámbito más característico de la información comunitaria: la normativa de la CE, así como el proceso asociado de adopción de la misma. En general, todo lo referente a aquellos documentos (normas, acuerdos interinstitucionales, concursos públicos, propuestas, etc.) que se hacen públicos a través del Diario Oficial de las Comunidades Europeas ( $D O C E$ ), según lo establecido por el artículo 191 CEE. 
Conocida por todos los profesionales del sector, utilizada por la mayoría de ellos y soportada en distribuidores de bases de datos de todos los paises comunitarios, CELEX (Comunitatis Europeae Lex) ha sido y es la herramienta de trabajo habitual para "navegar» en el extenso mar de la legislación comunitaria. Este sistema, nacido en 1971, ha venido cubriendo con todo tipo de aciertos y, digámoslo también, con ciertas limitaciones, las necesidades más inmediatas en lo que a información normativa se refiere. Consideramos, no sin fundamento, que se puede afirmar que CELEX responde con suficiente precisión al conocimiento de la legislación comunitaria aplicable en cada sector de actividad. Ello responde a lo que nos parecen dos importantes aciertos en su diseño. Por una parte, al tratarse de una base de datos en texto completo (incluido el texto íntegro de los Tratados), permite realizar búsquedas de información extremadamente especificas. Por otra parte, el método de codificación de todos los documentos que entran en la base de datos ha permitido la creación de un sistema de referencias cruzadas entre los documentos normativos que facilita al máximo la recuperación de toda la legislación relacionada con una norma específica que se haya publicado anterior o posteriormente a la misma. En fin, podemos decir que los 125.000 registros de documentación que hoy en día conviven "amigablemente» en CELEX, pueden dar respuesta, hábilmente manejados, a un sinfin de demandas sobre la legislación europea aplicable en un momento determinado y a un campo concreto.

No obstante, CELEX tiene, a nuestro parecer, dos grandes limitaciones. Por un lado, la actualización de sus ficheros es demasiado lenta (entre 30 y 45 días después de la publicación oficial del documento) para satisfacer a ciertos sectores que requieren una mayor rapidez y cobertura temporal de la información. Por otro lado, el diseño de la base de datos no se ajusta al modelo que sería necesario para facilitar el seguimiento de las distintas etapas que transcurren en la toma de decisiones comunitarias, especialmente en relación con los nuevos procedimientos establecidos a partir del Acta Unica: el procedimiento de cooperación y el de dictamen conforme.

En los dos últimos años, como ya se había comentado, han surgido sistemas de información que vienen, precisamente, a cubrir las lagunas anteriormente descritas. La Oficina de Publicaciones Oficiales de la CE (EUR-OP) ha querido contribuir a una mejor difusión de la normativa comunitaria, poniendo en marcha el sistema ABEL (Amtsblatt Elektronisch). Este sistema es el resultado de la combinación de una base de datos que recoge la referencia de la normativa comunitaria en el mismo día de su publicación y de un sistema de archivo óptico (ARCDON) que permite recuperar electrónicamente los textos originales de dicha normativa y enviarlos al usuario mediante cualquier sistema de transmisión electrónica (habitualmente telefax). Nos encontramos, por tanto, con la posibilidad de conocer y leer en texto completo cualquier documento legislativo de la CE a partir de la fecha de su publicación, sin necesidad de esperar ni la llegada del $D O C E$ impreso, ni la inclusión del registro documental en CELEX.

En el segundo de los ámbitos en que CELEX muestra su insuficiencia para una correcta cobertura de la información, es decir, en el seguimiento del proceso de toma de decisiones, el Parlamento Europeo ha diseñado y ha puesto en explotación su propio sistema de información denominado EPOQUE (European Parliament 
On-line Query). Este sistema incorpora, entre otras funcionalidades que no vamos a detallar ahora, un capitulo dedicado al desarrollo de los procesos normativos. A través del mismo, se puede consultar en qué etapa de discusión se encuentra cualquiera de las propuestas legislativas de la Comisión Europea, así como la referencia a todos los documentos asociados a este proceso. Evidentemente, el sistema es de especial interés en todos aquellos procesos normativos que se rigen por el procedimiento de cooperación (sistema de doble lectura por el Parlamento Europeo) y lo será aún más para el nuevo procedimiento previsto por el Tratado de Maastricht, denominado por el momento "procedimiento de codecisión", que puede implicar hasta una tercera lectura en la cámara parlamentaria europea. Si consideramos que el sistema EPOQUE es alimentado diariamente, es obvio que la actualidad de la información contenida en sus registros es inmediata.

Otra buena herramienta para el seguimiento de ciertos procesos normativos es el sistema INFO-92. Puesto en marcha por la Comisión Europea en junio de 1989, este sistema, basado en una organización de estructura arbórea de la información (no es una base de datos en el sentido tradicional), ha permitido y permite el seguimiento del estado de desarrollo de las 282 propuestas de legislación contenidas en el Libro Blanco para la realización del Mercado Unico Europeo. Evidentemente, dado que el plazo límite para la adopción de esta normativa venció el 31 de diciembre de 1992, este sector perderá gran parte de su actualidad para conservar un interés marcadamente histórico. Por ello, se ha incluido desde el pasado año un capítulo complementario que pretende cubrir la normativa relacionada con la dimensión social del mercado interior, es decir, con la Carta europea de los derechos sociales fundamentales de los trabajadores de 1989. Dicha Carta se ha incorporado en el Tratado de Maastricht en forma de protocolo junto a un acuerdo que ha sido adoptado por once de los doce Estados miembros (todos excepto el Reino Unido).

Por último, podriamos considerar como fuentes complementarias de información otras bases de datos que de una manera u otra referencian los actos normativos o los documentos que sirven para su preparación: SCAD (Système Communautaire d'accès à la Documentation) que incluye referencias bibliográficas no sólo de los documentos de la CE, sino también las procedentes del vaciado de más de 1.500 publicaciones especializadas; y RAPID (Comunicados de Prensa del Portavoz de la Comisión) cuyos textos resumen las más actuales actividades y propuestas normativas de esa institución.

Por todo ello, consideramos que, especialmente en los últimos años, la labor realizada por las instituciones comunitarias para facilitar el acceso público a su información es sustancial y abundante. Al menos, es suficiente para despejar cualquier acusación de oscurantismo o duda sobre la falta de interés por poner a disposición pública cualquier información derivada de sus actividades. Recordemos que, además, se mantienen oficinas de representación de la Comisión y del Parlamento Europeo en todas las capitales de los Estados miembros, con el fin de facilitar los medios suficientes para el acceso a los citados sistemas de información.

Otra cuestión, a nuesto entender, es la dificultad que entraña, para los profesionales de la documentación, el tener que manejar los sistemas de información descritos: pluralidad de lenguas de trabajo ( 9 lenguas oficiales); programas de 
tratamiento y gestión de bases de datos diferentes (derivados muchas veces de la multiplicidad de fabricantes europeos de ordenadores); ausencia de uso de herramientas documentales comunes (por ejemplo, sistemas de indización basados en diferentes tesauros); solapamiento de la información contenida en las bases de datos; etc.

En estos puntos es donde sí creemos necesario un esfuerzo por homogeneizar y armonizar los sistemas. Si bien la tarea no es fácil, pues se requiere para ello un alto grado de coordinación interdepartamental e interinstitucional, este esfuerzo se vería ampliamente compensado por el beneficio obtenido de cara al usuario final y por el ahorro de algunos de los recursos que actualmente se emplean en acciones paralelas.

La extensión del uso de EUROVOC como herramienta común para la indización de los documentos; la utilización del CCL (Common Command Language), sin adaptaciones ni retoques, como lenguaje de consulta para todas las bases de datos; la carga de todos los sistemas de información sobre un solo distribuidor de bases de datos, etc., podrian ser algunas de las acciones encaminadas a facilitar esa ansiada armonización de los sistemas de tratamiento de la información comunitaria, que todos deseamos.

Por tanto, cuando leemos en el Tratado de la Unión Europea, la declaración relativa al derecho de acceso a la información: "La Conferencia estima que la transparencia del proceso de decisión refuerza el carácter democrático de las Instituciones, así como la confianza del público en la Administración...», concluimos decididamente que los sistemas de información actualmente en servicio son suficientes, tanto en su cantidad como en su calidad, para dar sobrado cumplimiento a este mandato, si bien, como ya se ha indicado anteriormente, una mayor armonización de los mismos facilitaría, aún más, el objetivo final: una total e inmediata transparencia informativa. En cualquier caso, el Consejo Europeo, en el texto de la citada declaración, invita a la Comisión a presentar, en 1993, un informe sobre medidas encaminadas a «mejorar el acceso del público a la información de que disponen las Instituciones».

\section{Leer Maastricht en clave de documentalista}

Muchas son las lecturas que pueden realizarse del Tratado de Maastricht. Cada profesional, cada técnico, cada ciudadano, encontrará importantes novedades en los contenidos de un texto que, a pesar de su complejidad, revela claramente las líneas del desarrollo de la futura Unión Europea. Ya comentábamos, en la primera parte, que los contenidos de dicho Tratado constituyen un sustancial avance con respecto a la reforma que supuso el Acta Unica Europea.

Nuestra modesta aportación a la interpretación del Tratado se basa en la lectura desde una óptica profesional. ¿Qué novedades podrán derivarse de la aplicación de los principios y de las normas derivadas de Maastricht? ¿Qué adaptaciones serán necesarias en los sistemas de información actuales' Trataremos de exponerlo. 
En primer lugar debemos llamar la atención sobre una cuestión terminológica: desaparece la Comunidad Económica Europea.

En adelante, no deberá ser utilizado más dicho término ni, lógicamente, sus siglas CEE. El Tratado de Maastricht rebautiza todo el Tratado CEE como «Tratado de la Comunidad Europea». Técnicamente, consideramos que se deberá seguir hablando de "Comunidades Europeas», en todas aquellas referencias a instituciones y órganos comunes a los tres tratados (el mencionado, más los tratados CECA y Euratom). Sin embargo, este cambio terminológico, facilitará en gran medida la referencia a políticas comunes de la "Comunidad Europea» que van más allá del simple ámbito económico al que anteriormente parecían restringidas dada la denominación del tratado de que derivaban.

Desde el punto de vista de la documentación normativa, Maastricht implica varias novedades importantes: Un nuevo procedimiento de toma de decisiones, a añadir a los ya existentes, la creación de un nuevo órgano consultivo: el Comité de las Regiones; la modificación del artículo $191 \mathrm{CEE}$, que definía las fórmulas de publicidad de los actos juridicos y un mandato para la revisión de la jerarquía de los actos comunitarios.

En lo que se refiere al nuevo procedimiento de toma de decisiones, al que denominaremos «procedimiento de codecisión», el Tratado establece que, para ciertas materias específicas como la libre circulación de trabajadores, el mercado interior, la investigación, el medio ambiente, la protección de los consumidores, las redes transeuropeas, la educación, la cultura y la salud pública, el Consejo y el Parlamento Europeo deberán adoptar conjuntamente las normas comunitarias. Ello no implica, en absoluto, la desaparición de los cuatro procedimientos tradicionales que se mantienen vigentes: consulta; cooperación; dictamen conforme y procedimiento presupuestario.

El procedimiento de codecisión establece un complejo mecanismo por el cual la Comisión Europea seguirá teniendo el poder de iniciativa legislativa, pero la redacción final del texto normativo ya no será aprobada en solitario por el Consejo de Ministros, sino que deberá ser "pactada» con el Parlamento Europeo en el seno de un Comité de Conciliación, y ratificada por ambas instituciones para convertirla en una norma comunitaria. Este mecanismo dará origen a nuevos tipos documentales: textos comunes derivados del Comité de Conciliación; textos de la Comisión con propuestas y dictámenes que acompañen al procedimiento; informes y resoluciones del Parlamento Europeo para las terceras lecturas (lecturas de ratificación), etcétera. Qué duda cabe que, además de los nuevos tipos de documentos, este procedimiento implicará una reforma de los sistemas de información automatizada que, hoy en día, permiten el seguimiento del proceso de adopción de normas. Nuevas notaciones del tipo SYN que hoy se utiliza para los procedimientos de cooperación deberán incluirse también en todos los documentos asociados al mismo procedimiento de codecisión. En definitiva, cada vez será más importante el uso de sistemas automatizados de información que permitan realizar un adecuado seguimiento de los procesos normativos comunitarios, pues, dada la complejidad creciente que se les ha ido dando, será ésta la mejor manera de conocer la evolución de los textos normativos a lo largo de su discusión. 
El Comité de las Regiones será un nuevo órgano consultivo para la elaboración de la legislación comunitaria en todo aquello que afecte a la política regional o cuando así lo soliciten el Consejo o la Comisión. Su composición numérica será similar a la del actual Comité Económico y Social (CES), y sus miembros representarán a los entes regionales y locales de los doce países comunitarios. En cuanto a su organización funcional, seguirá básicamente las orientaciones del actual funcionamiento del CES. Sus dictámenes constituirán, por tanto, una nueva serie documental de la $\mathrm{CE}$.

El actua! artículo $191 \mathrm{CEE}$ establece las normas de publicidad de los actos jurídicos comunitarios: los reglamentos se publicarán en el $D O C E$ y surtirán efecto a los 20 días de su publicación; las directivas y las decisiones se notificarán a sus destinatarios y surtirán efecto a partir de dicha notificación (no es necesaria su publicación en el $D O C E$ ). Este artículo será modificado sustancialmente cuando entre en vigor el Tratado de Maastricht. El nuevo artículo $191 \mathrm{CE}$ establece varios cambios. En primer lugar, todos los actos jurídicos (reglamentos, directivas y decisiones) adoptados por el procedimiento de codecisión serán obligatoriamente publicados en el DOCE y entrarán en vigor, salvo que se fije lo contrario, a los 20 días de su aparición. Además de los reglamentos, cuya publicación ya era obligatoria, las directivas que tengan como destinatarios a todos los Estados miembros, también serán de obligada publicación en el $D O C E$. Este aspecto no hace más que consolidar un hábito de publicidad que ya existía, aunque no fuese consecuencia de la obligatoriedad jurídica. Para el resto de directivas y para todas las decisiones, bastará la notificación a los interesados para que surtan efecto.

Por último, en materia normativa el Tratado de Maastricht establece, en su declaración relativa a la jerarquía de los actos comunitarios, que la Conferencia Intergubernamental que deberá convocarse en 1996, debe estudiar una posible revisión de la clasificación de los actos comunitarios, con el fin de establecer una adecuada jerarquía entre las distintas categorías de normas. Esto significa que, por el momento, seguiremos trabajando sobre una reducida tipología de normas: los reglamentos, las directivas y las decisiones.

No obstante, la inclusión en el Tratado de la citada Declaración nos advierte sobre el espíritu de una próxima reforma de la nomenclatura y de la jerarquia de los actos jurídicos comunitarios.

«Se crea una ciudadanía de la Unión. Será ciudadano de la Unión toda persona que ostente la nacionalidad de un Estado miembro.» (Nuevo artículo 8.1 CE). Con este nuevo texto, el Tratado de Maastricht introduce un catálogo de derechos que deberán ser reconocidos a todos los ciudadanos que formen parte de la Unión: libertad de circulación y residencia; sufragio activo y pasivo en elecciones locales y europeas; derecho a la protección consular; derecho de petición ante el Parlamento Europeo y derecho de protección del Defensor del Pueblo Europeo (creado por el nuevo art. 138 E). En fin, un primer conjunto de "señas de identidad" para los ciudadanos europeos, que podrá ser ampliado en un futuro si la unanimidad del Consejo así lo desea.

La definición de una nueva figura, la del Defensor del Pueblo, implicará, evidentemente, la aparición de nuevas series documentales derivadas de su actividad (por ejemplo, cada año deberá presentarse al Parlamento Europeo un informe 
sobre los resultados de las investigaciones del Defensor del Pueblo). Creemos que los nuevos derechos reconocidos en Maastricht, en lo que se refiere a la petición ante el Parlamento Europeo y a la reclamación ante el Defensor del Pueblo, deberán dar origen a sistemas de información pública que permitan conocer cuál es el estado de tramitación de dichos procedimientos administrativos. De hecho, el Parlamento Europeo, en su base de datos EPOQUE, ya incluye el seguimiento de las actuales peticiones que recibe de los ciudadanos.

La Unión Económica y Monetaria (UEM) es otra de las grandes novedades del Tratado. Evidentemente, las consecuencias económicas y monetarias de la misma son extensísimas y constituyen uno de los núcleos centrales del nuevo Tratado. Sin entrar a analizar las implicaciones que supone la existencia de una moneda única europea y del seguimiento que se deberá realizar de las economías de los Estados miembros en sus fases de convergencia hacia la misma, sí consideramos interesante señalar que de la construcción de la Unión Económica y Monetaria se derivará la aparición de nuevas instituciones europeas: el Comité Monetario (que dará origen a un Comité Económico y Financiero en la tercera fase de la UEM); el Instituto Monetario Europeo (IME) que se creará en la segunda fase de la UEM (1 de enero de 1994) para asumir las funciones del FECOM (Fondo Europeo de Cooperación Monetaria); y, por último, el Banco Central Europeo, que, además de sus competencias institucionales, estará dotado de poder normativo. En efecto, el BCE elaborará los reglamentos y adoptará las decisiones que sean necesarias para el ejercicio de las funciones encomendadas al SEBC (Sistema Europeo de Bancos Centrales, compuesto por el BCE y los bancos centrales de cada uno de los estados miembros). Asimismo, podrá emitir recomendaciones y dictámenes que no serán vinculantes. Tanto los reglamentos como las decisiones del BCE tendrán las mismas implicaciones en cuanto a su publicidad y a su efecto jurídico, que los correspondientes actos normativos comunitarios ya tipificados actualmente.

Por todo ello, la derivación institucional y normativa de la UEM tiene una gran amplitud e importantes consecuencias para el seguimiento documental de la aplicación del Tratado de Maastricht.

En lo que se refiere a las políticas comunes, el Tratado aporta importantes novedades, especialmente por la inclusión de nuevos ámbitos de actuación de la Comunidad Europea: cultura, salud pública, protección de los consumidores, redes transeuropeas, educación, formación profesional y juventud. Asimismo, refuerza la acción comunitaria en los temas referentes a medio ambiente, investigación y cooperación al desarrollo. Los efectos de dichos cambios, desde el punto de vista de los sistemas de información, no nos parecen importantes. Cabe esperar que, una vez que la CE haya asumido estas competencias, se refuercen y mejoren los servicios de información en las áreas que ya existian, y que aparezcan nuevos sistemas que faciliten un mejor conocimiento de la realidad europea en las áreas de actuación más recientes.

Por último, los títulos V y VI del Tratado de Unión Europea establecen las disposiciones relativas a la PESC (Política Exterior y de Seguridad Común) y a la cooperación en los ámbitos de la Justicia y de los asuntos de Interior. Ambos apartados establecen las bases de una cooperación intergubernamental en las 
citadas materias. No se trata, por tanto, de la definición de políticas comunes, sino de la creación de mecanismos de cooperación que permitan a los estados miembros presentar y mantener posiciones comunes para coordinar su acción. Cabe resaltar, en materia de seguridad, que se encomienda a la UEO (Unión Europea Occidental) que «elabore y ponga en práctica las decisiones y acciones de la Unión que tengan repercusiones en el ámbito de la defensa». Por el momento será la UEO, la encargada de coordinar las cuestiones de la defensa europea, a la espera, en el futuro, de la definición de una política de defensa común (art. J.4).

Por otra parte, de la cooperación en materia policial podrá surgir el germen de una organización paneuropea que aglutine los esfuerzos de los sistemas de información policiales de cada Estado miembro: la Europol.

No obstante, insistimos en la ausencia de consecuencias directas sobre las fuentes documentales de estos mecanismos de cooperación intergubernamental, dado que su actuación no tiene apenas incidencia ni institucional, ni normativa.

\section{Consideraciones finales}

A lo largo de la presente exposición sobre el Tratado de Maastricht, hemos intentado dar respuesta a dos cuestiones:

- ¿Están los sistemas de documentación de la CE lo suficientemente desarrollados para asegurar la publicidad y la transparencia de su información hacia los ciudadanos?

- ¿Cuáles son los elementos más importantes a tener en cuenta del Tratado, en relación a su incidencia en los sistemas de información?

Creemos que a la primera de ellas es posible contestar con una afirmación rotunda la riqueza actual de recursos de información comunitaria es suficiente para asegurar el rápido y preciso conocimiento de cualquiera de los documentos que son tratados por las instituciones de la CE. Quizás en el esfuerzo por crear los servicios y ponerlos a disposición pública se han quedado en la cuneta algunas cuestiones relacionadas con la homogeneización y la armonización de los propios sistemas de información. La utilización de tesauros comunes, la aplicación de lenguajes documentales más uniformes, el uso de un distribuidor de bases de datos único, etc., pueden ser los caminos para resolver los problemas que provoca la actual dispersión. A pesar de todo ello, no faltan fuentes de información con las que poder trabajar para resolver la mayor parte de necesidades de los servicios de documentación.

En lo referente a las implicaciones del Tratado de Maastricht sobre los sistemas documentales, hemos intentado señalarlas a lo largo de la exposición de cada uno de los temas abordados por el Tratado. Creemos que, en general, los cambios son importantes. No sólo porque constituyen un paso sustancial en la construcción europea, sino porque ello implica la aparición de reformas institucionales y normativas muy relevantes. Cuatro nuevas instituciones se incorporan a la vida diaria de la $\mathrm{CE}$; un nuevo y complejo procedimiento de toma de decisiones se define; los 
ciudadanos tendrán más y mejor acceso a la administración comunitaria; nuevas políticas se integran en las competencias comunitarias, etc. Todo ello conllevará, seguro, no sólo una importante adaptación de los sistemas de información sino también un notable aumento en la extensión de los ámbitos de sus contenidos.

Evidentemente, esto significará también un esfuerzo de formación y adaptación de los profesionales documentalistas, que se verán obligados a conocer y comprender desde los nuevos tipos documentales hasta los nuevos procedimientos de adopción de las normas comunitarias.

La Unión Europea asi lo exige. Sólo el esfuerzo y la imaginación harán factibles los progresos necesarios hacia la realidad europea del futuro. 